1 Department of General Practice, Amsterdam Public Health, Amsterdam University Medical Centers, University of Amsterdam, 9 Meibergdreef, PO Box 22660, 1100 DD Amsterdam, Netherlands

2 Department of Family Medicine, Care and Public Health Research Institute, Faculty of Health, Medicine, and Life Sciences, Maastricht University, Netherlands

3 Department of Methodology and Statistics, Care and Public Health Research Institute, Faculty of Health, Medicine, and Life Sciences, Maastricht University, Netherlands

4 Netherlands Institute for Health Services Research, Utrecht, Netherlands

5 Department of Health Services Research, Care and Public Health Research Institute, Faculty of Health, Medicine, and Life Sciences, Maastricht University, Netherlands

Correspondence to: S B Uittenbogaart s.b.uittenbogaart@amsterdamumc.nl http://orcid.org/0000-0002-9798-6472 Cite this as: BMJ 2020;370:m3208 http://dx.doi.org/10.1136/bmj.m3208 Accepted: 07 August 2020 Published: 16 September 2020

\title{
Opportunistic screening versus usual care for detection of atrial fibrillation in primary care: cluster randomised controlled trial
}

\author{
Steven B Uittenbogaart, ${ }^{1}$ Nicole Verbiest-van Gurp, ${ }^{2}$ Wim A M Lucassen, ${ }^{1}$ Bjorn Winkens, ${ }^{3}$ Mark Nielen, \\ Petra M G Erkens, ${ }^{5}$ J André Knottnerus, ${ }^{2}$ Henk C P M van Weert, ${ }^{1}$ Henri E J H Stoffers ${ }^{2}$
}

\section{ABSTRACT \\ OBJECTIVE}

To investigate whether opportunistic screening in primary care increases the detection of atrial fibrillation compared with usual care.

\section{DESIGN}

Cluster randomised controlled trial.

\section{SETTING}

47 intention-to-screen and 49 usual care primary care practices in the Netherlands, not blinded for allocation; the study was carried out from September 2015 to August 2018.

\section{PARTICIPANTS}

In each practice, a fixed sample of 200 eligible patients, aged 65 or older, with no known history of atrial fibrillation in the electronic medical record system, were randomly selected. In the

intention-to-screen group, 9218 patients eligible for screening were included, $55.0 \%$ women, mean age 75.2 years. In the usual care group, 9526 patients were eligible for screening, $54.3 \%$ women, mean age 75.0 years.

\section{INTERVENTIONS}

Opportunistic screening (that is, screening in patients visiting their general practice) consisted of three index tests: pulse palpation, electronic blood pressure measurement with an atrial fibrillation algorithm, and electrocardiography (ECG) with a handheld single lead electrocardiographic device. The reference standard was 12 lead ECG, performed in patients with at least one positive index test and in a sample of patients (10\%) with three negative tests. If 12 lead ECG showed no atrial fibrillation, patients were invited for more screening by continuous monitoring with a Holter electrocardiograph for two weeks.

\section{MAIN OUTCOME MEASURES}

Difference in the detection rate of newly diagnosed atrial fibrillation over one year in intention-to-screen versus usual care practices.

\section{RESULTS}

Follow-up was complete for 8874 patients in the intention-to-screen practices and for 9102 patients in the usual care practices. 144 (1.62\%) new diagnoses of atrial fibrillation in the intention-to-screen group versus 139 (1.53\%) in the usual care group were found (adjusted odds ratio 1.06 (95\% confidence interval 0.84 to 1.35)). Of 9218 eligible patients in the intention-to-screen group, 4106 (44.5\%) participated in the screening protocol. In these patients, 12 lead ECG detected newly diagnosed atrial fibrillation in 26 patients (0.63\%). In the 266 patients who continued with Holter monitoring, four more diagnoses of atrial fibrillation were found.

\section{CONCLUSIONS}

Opportunistic screening for atrial fibrillation in primary care patients, aged 65 and over, did not increase the detection rate of atrial fibrillation, which implies that opportunistic screening for atrial fibrillation is not useful in this setting.

TRIAL REGISTRATION

Netherlands Trial Register No NL4776 (old NTR4914).

\section{Introduction}

Atrial fibrillation is a common cardiac arrhythmia and a major cause of stroke, heart failure, and other cardiovascular diseases. ${ }^{1}$ The prevalence of atrial fibrillation increases with age, and with the ageing population, the burden of atrial fibrillation is growing rapidly. ${ }^{23}$ Treatment with oral anticoagulant drugs reduces the risk of stroke by $60 \%$. In $25 \%$ of patients who have had a stroke, however, atrial fibrillation is not diagnosed until after the stroke. ${ }^{4}$

Patients with atrial fibrillation might present with symptoms such as palpitations, shortness of breath, light headedness, or dizziness. Physicians can detect an irregular heart rhythm by physical examination and can confirm atrial fibrillation with 12 lead electrocardiography (ECG). ${ }^{5}$ But the possible paroxysmal character of atrial fibrillation and the occasional asymptomatic course of the condition can hamper detection. ${ }^{6}$

Screening for atrial fibrillation could increase early detection and subsequent treatment of atrial fibrillation, and prevent strokes, but community screening for atrial fibrillation is still controversial. ${ }^{78}$ Randomised trials comparing the results of screening with usual care are lacking. Trials evaluating the effect of treating atrial fibrillation detected by screening with oral anticoagulant agents are pending. The Screening for Atrial Fibrillation in the Elderly (SAFE) study is the only randomised controlled trial that has compared screening, by pulse palpation, with usual care, in a primary care population. ${ }^{9}$ Both systematic (inviting the whole target population) and opportunistic (only screening patients who visited the practice) screening detected more new diagnoses than usual care (yearly incidence of atrial fibrillation $1.62 \%$ and $1.64 \% v 1.04 \%$, respectively). The investigators preferred opportunistic screening because of the more labour intensive, costly, and intrusive approach of systematic screening.

Since the publication of the SAFE study in 2007, numerous devices have been developed to screen for atrial fibrillation (eg, electronic blood pressure monitors with an atrial fibrillation detection function and handheld single lead electrocardiographic devices). ${ }^{10}$ We performed the Detecting and 
Diagnosing Atrial Fibrillation (D2AF) study to investigate whether opportunistic screening in primary care patients increased the detection of atrial fibrillation compared with usual care. ${ }^{11}$ Three methods were used to detect atrial fibrillation: pulse palpation, electronic blood pressure measurement with an atrial fibrillation algorithm, and ECG with a handheld single lead electrocardiographic device. Also, we explored the added value of continuous Holter monitoring for two weeks.

\section{Methods}

\section{Trial design}

We performed a cluster randomised controlled trial comparing opportunistic screening of atrial fibrillation with usual care. The study was carried out for one year in each practice, after which we compared the number of new diagnoses of atrial fibrillation in the intention-to-screen with the usual care practices. We published the study protocol previously. ${ }^{11}$

\section{Practices and randomisation}

We recruited primary care practices, located throughout the Netherlands, within the networks of the two participating universities and the Dutch Nivel Primary Care Database. ${ }^{12}$ We used computerised randomisation in permuted blocks of random sizes (four, six, and eight). We stratified on region (north or south) and the pre-existing prevalence of atrial fibrillation in all patients in the practice aged 65 and over (the cut-off was $8.05 \%$, based on the prevalence of atrial fibrillation in 2015 in a group of primary care practices associated with the two universities conducting this study). We used the pre-existing prevalence of atrial fibrillation as a marker of the risk of atrial fibrillation in the practice population and the awareness of the general practitioner in detecting atrial fibrillation. Practices were not blinded for allocation.

\section{Selected patients}

In each practice, we randomly selected and marked a fixed sample of 200 patients eligible for opportunistic screening, aged 65 or older, with no known history of atrial fibrillation in the electronic medical record system. We used the International Classification of Primary Care codes to exclude patients with a history of atrial fibrillation (K78) and to determine baseline information on relevant morbidities. ${ }^{13}$

\section{Intention-to-screen practices}

We provided practices with an electronic blood pressure monitor with an atrial fibrillation detection function (WatchBP Home A, Microlife, Widnau, Switzerland), two handheld single lead electrocardiographic devices (MyDiagnostick, MyDiagnostick Medical, Maastricht, Netherlands), and ECG and Holter equipment (multichannel Holter electrocardiograph recorder model $\mathrm{H}_{2}$, Fysiologic, Amsterdam, Netherlands). The secondary investigators (practice nurse, practice assistant, general practitioner) were trained on the use of the study software and equipment and given instructions on pulse palpation. We instructed practices to ask eligible patients to participate in the study when they visited the practice in the study year.

When the physician (or other practice staff) opened the electronic medical record of a marked patient, a notification on their computer screen alerted them that the patient was selected for screening for atrial fibrillation. Patients were not eligible if they had a pacemaker or an implantable cardioverter defibrillator, could not provide informed consent, had a terminal illness, or could not visit the practice.
After obtaining written consent, the secondary investigator collected information (at that visit or at a later visit) on symptoms related to atrial fibrillation and performed the three index tests: palpation of the radial pulse was always performed first, followed by an electronic blood pressure monitor with an atrial fibrillation detection function, and ECG with the handheld device, in a preset alternating order. We instructed the secondary investigators to palpate the radial pulse for a minimum of 15 seconds; any irregularity was regarded as a positive test. For both electronic devices, we used the automated algorithm for detection of atrial fibrillation: a blinking AFIB icon on the electronic blood pressure monitor and a red indicator light on the device.

Patients with at least one positive index test, and a random sample of patients ( $10 \%$; generated by the study software) with three negative index testsunderwent 12 lead ECG as the reference standard for atrial fibrillation. We instructed the investigators to perform 12 lead ECG immediately after the index tests. The results of the ECG were transferred digitally and evaluated by an experienced assessor, supervised by a cardiologist. A second cardiologist re-evaluated all electrocardiograms. In the event of disagreement, a third cardiologist decided on the diagnosis. The general practitioner's office received the electrocardiogram and a report of the assessment by the cardiologist.

We invited patients in whom 12 lead ECG showed no atrial fibrillation to undergo continuous Holter recording for two weeks.

\section{Usual care practices}

In the usual care practices, the secondary investigators and patients were unaware of which 200 patient records were selected. During recruitment, we informed all practices about the aim of the study but did not provide equipment or training. During the study year, the practice could not participate in other screening activities related to atrial fibrillation.

\section{Usual care in the Netherlands}

The guideline for atrial fibrillation from the Dutch College of General Practitioners recommends assessing heart rhythm in every patient with shortness of breath, reduced ability to exercise, palpitations, dizziness, light headedness, syncope, chest pain, and transient ischaemic attack or stroke, as part of the usual diagnostic work-up. Further recommendations are to assess the heart rhythm in each patient when measuring blood pressure. ${ }^{14}$ Systematic screening is not recommended. In the past few years, structured disease management programmes were introduced in Dutch general practice. Patients with a cardiovascular disease, diabetes, or chronic respiratory disease, or with a risk factor for these diseases, could participate in the programmes and visit the practice at least once a year. During these visits, the heart rhythm was assessed with pulse palpation or sometimes with an electrocardiograph. ${ }^{15}$

\section{Diagnosis of atrial fibrillation}

Three months after the end of the study year, in the intention-to-screen and usual care practices, we extracted the International Classification of Primary Care codes for atrial fibrillation and related diagnoses (that is, palpitations, irregular pulse, paroxysmal tachycardia, extrasystoles, transient ischaemic attack, and stroke) from the electronic medical records of the marked patients. We accepted a diagnosis of atrial fibrillation if it was confirmed by ECG, in primary or secondary care. We also accepted a description of the diagnosis of atrial fibrillation in a hospital letter. We only included diagnoses within the study year. If a diagnosis preceded the start of the study, we excluded these patients from the analyses (eg, if the diagnosis of atrial fibrillation was miscoded 
in the electronic medical record or if a hospital letter with a diagnosis of atrial fibrillation preceding the start of the study arrived late). Follow-up was incomplete if the patient died, or if they unregistered or moved away from the practice during the study year.

\section{Outcome and sample size}

Our primary outcome was the difference in the number of patients with newly diagnosed atrial fibrillation during the study year between the intention-to-screen and usual care practices. We based our sample size on a yearly incidence of new atrial fibrillation of $1.3 \%,{ }^{16}$ a minimum detectable odds ratio of 1.8 of identifying new atrial fibrillation by opportunistic screening versus usual care, and $80 \%$ power at a significance level of $5 \% .9^{1718}$ Given a calculated design effect of 2.99 based on fixed cluster sizes of 200 patients and an intracluster correlation coefficient of $0.01,{ }^{19}{ }^{20}$ a minimum of 8076 patients in each study arm was required. We corrected for a loss to follow-up of $15 \%$. Thus 96 clusters of 200 patients were required, a total of 19200 patients.

\section{Statistical methods}

We assessed the difference in detection rate of atrial fibrillation with logistic mixed effects models with a random intercept on general practice to account for clustering of patients within a practice. For the intention-to-screen analysis, the fixed part of the model consisted of group (intention to screen $v$ usual care) and stratification variables (that is, prevalence of atrial fibrillation and region). For the sensitivity analysis, we performed multiple imputation of missing outcome data where the missing outcome was imputed with group, age, sex, and stratification variables. After the creation of five complete datasets by multiple imputation with 20 iterations, we compared the pooled results with the original analysis.

In an ad hoc per protocol analysis, we compared patients who were screened with patients who received usual care. We performed similar analyses but we also corrected for potential confounders: age (in years), sex (male or female), and history of hypertension, diabetes mellitus, stroke (transient ischaemic attack and stroke), thromboembolism, and heart failure. Also, we assessed whether atrial fibrillation was detected earlier in the intention-to-screen group than in the usual care group, by performing a survival analysis (Kaplan-Meier curves, log rank tests, and Cox regression analysis with the same fixed variables). In this sensitivity analysis, (censored) time to atrial fibrillation was defined as the difference between inclusion date and date of detection of atrial fibrillation, death, lost to follow-up, or end of the study year, whichever occurred first.

Continuous variables are reported as mean (standard deviation), and categorical variables as numbers and percentages. A two sided $P$ value of 0.05 or less was considered statistically significant. We used IBM SPSS Statistics for Windows (version 25.0, IBM, Armonk, NY) for all analyses, except for the logistic mixed effects models, which we assessed with the glmer package with RStudio (version 1.2.5019, RStudio, Boston, MA).

\section{Patient and public involvement}

Patient influence on research design, methodology, and execution: none. At the time of the study design, the funding organisation did not request explicit public or patient involvement in clinical studies in general practice. But patient representatives participated in scientific committees that judged eligibility for funding.

\section{Results}

Initially, we recruited 97 general practices in the Netherlands, and randomisation resulted in 48 intention-to-screen and 49 usual care practices. One intention-to-screen practice withdrew directly after randomisation. Thus 96 primary care practices participated in the study. In one small practice, only 189 patients were potentially eligible, resulting in 19189 patients.

The study was carried out between September 2015 and August 2018. After data collection had ended, we detected 130 and 175 patients with a diagnosis of atrial fibrillation preceding the start of the study year that was not known at the time of selection in the intention-to-screen and usual care practices, respectively. We excluded these patients from the analyses. Thus 9218 and 9526 eligible patients in the intention-to-screen and usual care groups, respectively, were included in the analyses (fig 1 ). 


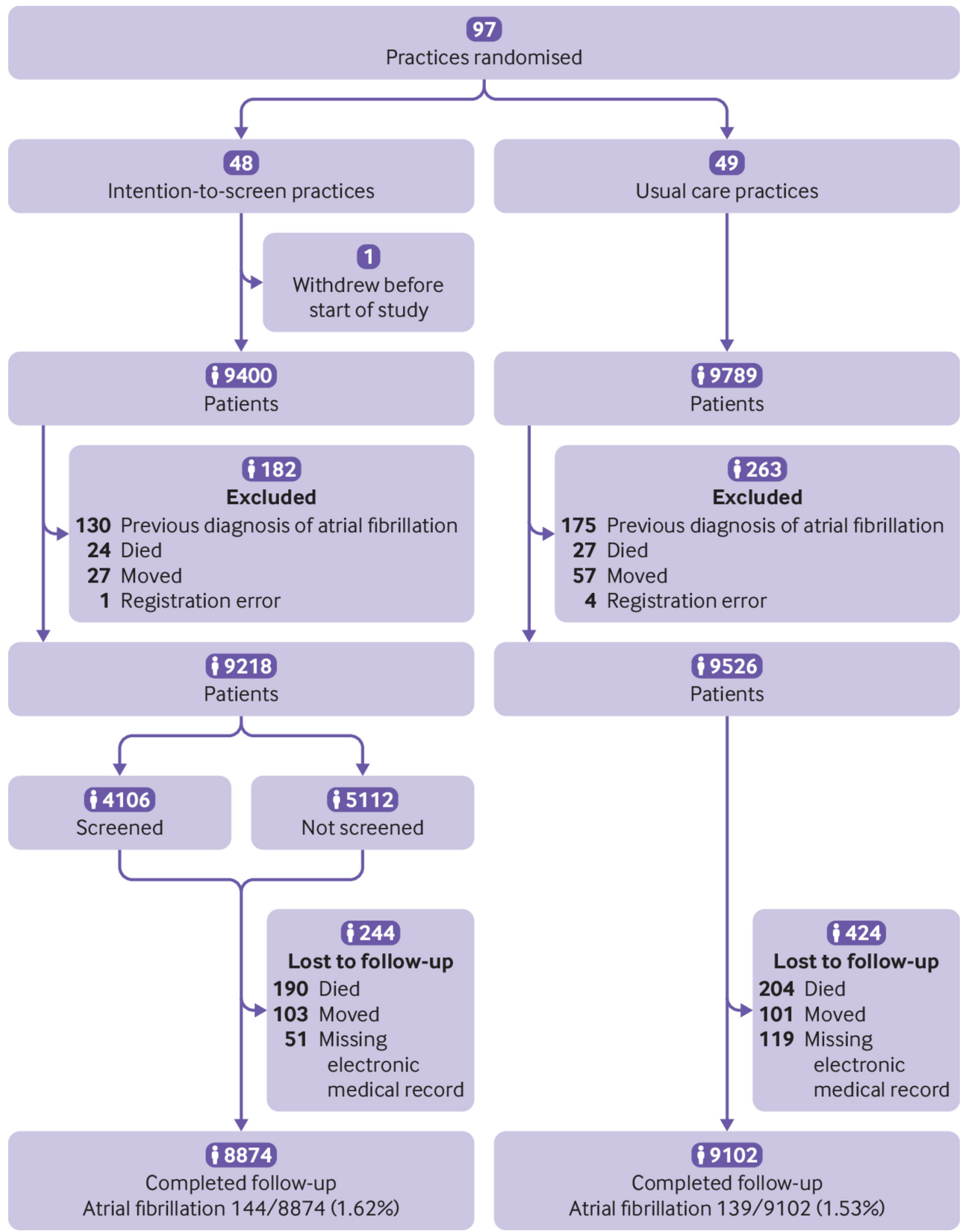

Fig 1 | Flowchart of enrolment of primary care practices (clusters) and patients in the study

\section{Baseline characteristics}

Baseline prevalence of atrial fibrillation in patients aged 65 or older was comparable in the intention-to-screen and usual care practices
(10.1\% and $10.0 \%$, respectively). The mean baseline prevalence of atrial fibrillation was higher than the stratification threshold of $8.05 \%$; in 83 of 96 practices, the prevalence was greater than $8.05 \%$. 
At baseline, mean age was 75.2 (standard deviation 6.8) years in the intention-to-screen group and 75.0 (6.9) years in the usual care, and most patients were women ( $55.0 \%$ and $54.3 \%$, respectively). Comorbidities were equally distributed across the groups (table 1). In the intention-to-screen group, the screened population was younger than those not screened $(73.5$ (5.5) years $v 76.6$ (7.3) years,
$\mathrm{P}<0.001)$, and the screened population had fewer comorbidities, including stroke and transient ischaemic attack $(7.7 \% \mathrm{v} 11.3 \%$, $\mathrm{P}<0.001)$ and heart failure $(1.8 \% v 5.4 \%, \mathrm{P}<0.001)$. Only hypertension was more frequent in the screened population (51.2\% $v 48.3 \%$, $\mathrm{P}=0.006)$.

Table 1 | Baseline characteristics of patients included in the Detecting and Diagnosing Atrial Fibrillation (D2AF) study

\begin{tabular}{|c|c|c|c|c|c|}
\hline & \multirow[t]{2}{*}{ Usual care } & \multicolumn{4}{|c|}{ Intention to screen } \\
\hline & & Total & Screened & Not screened & $P$ value* \\
\hline No & 9526 & 9218 & 4106 & 5112 & - \\
\hline Women (No (\%)) & $5177(54.3)$ & $5071(55.0)$ & $2196(53.5)$ & $2875(56.2)$ & $0.008 \ddagger$ \\
\hline Hypertension (No (\%)) & $4579(48.7)$ & $4540(49.6)$ & $2098(51.2)$ & $2442(48.3)$ & $0.006 \ddagger$ \\
\hline $\begin{array}{l}\text { Stroke or transient ischaemic } \\
\text { attack (No (\%)) }\end{array}$ & $911(9.7)$ & $886(9.7)$ & $315(7.7)$ & $571(11.3)$ & $<0.001 \neq$ \\
\hline Diabetes (№ (\%)) & $1750(18.6)$ & $1768(19.3)$ & $732(17.9)$ & $1036(20.5)$ & $0.002 \ddagger$ \\
\hline Heart failure (No (\%)) & $362(3.9)$ & $348(3.8)$ & $75(1.8)$ & $273(5.4)$ & $<0.001 \neq$ \\
\hline Thromboembolism (No (\%)) & $431(4.6)$ & $460(5.0)$ & $191(4.7)$ & $269(5.3)$ & $0.15 \ddagger$ \\
\hline
\end{tabular}

$\mathrm{SD}=$ standard deviation.

International Classification of Primary Care codes were used: for hypertension K86 or K87, or both; for stroke K90; for transient ischaemic attack K89; for diabetes T90; for heart failure K77; and for thromboembolism $\mathrm{K}_{93}$ or $\mathrm{K}_{94}$, or both.

For intention to screen and usual care, 62 patients (0.7\%) and $127(1.3 \%)$ had missing values for all comorbidities.

* Screened versus not screened in the intention-to-screen group.

† Independent sample t test.

₹ Pearson $\chi 2$ test.

\section{Intention to screen versus usual care}

Complete follow-up data were available for 8874 patients (96.3\%) in the intention-to-screen group and for 9102 patients (95.5\%) in the usual care group (fig 1). After one year, we found 144 (1.62\%) patients with newly diagnosed atrial fibrillation in the intention-to-screen group and $139(1.53 \%)$ in the usual care group (odds ratio 1.06, 95\% confidence interval 0.84 to 1.35 , corrected for clustering and stratification variables) (fig 1). In the per protocol group, (that is, those who were screened with the intervention protocol), we found 48 new diagnoses of atrial fibrillation, 26 detected by opportunistic screening, four by Holter monitoring, and 18 during usual care. We found an incidence of $1.18 \%$ in the per protocol group versus $1.53 \%$ in the usual care group (adjusted odds ratio $0.86,95 \%$ confidence interval 0.61 to 1.20 ). The results before and after multiple imputation were similar, and the Cox regression analysis showed no significant difference in time to detection of atrial fibrillation for the intention-to-screen and per protocol analyses (table 2).

Table 2 | Intention to screen versus usual care primary outcome and post hoc analyses

\begin{tabular}{|c|c|c|c|c|c|}
\hline Analysis & Group & $\begin{array}{c}\text { Intention to screen } \\
\text { (newly diagnosed atrial } \\
\text { fibrillation/all patients (No)) }\end{array}$ & $\begin{array}{c}\text { Usual care } \\
\text { (newly diagnosed atrial } \\
\text { fibrillation/all patients (No)) }\end{array}$ & $\begin{array}{l}\text { Odds ratio or hazard ratio } \\
\qquad(95 \% \mathrm{Cl})\end{array}$ & $P$ value \\
\hline Primary analysis & Intention to screen & $144 / 8874$ & 139/9102 & $1.06^{*}$ (0.84 to 1.35$)$ & 0.60 \\
\hline Primary analysis & Per protocol & $48 / 4085$ & 139/9102 & $0.86 *$ (0.61 to 1.20$)$ & 0.36 \\
\hline Multiple imputation & Intention to screen & $\mathrm{N} / \mathrm{A}$ & $\mathrm{N} / \mathrm{A}$ & $1.04^{*}(0.82$ to 1.31$)$ & 0.75 \\
\hline Multiple imputation & Per protocol & N/A & $\mathrm{N} / \mathrm{A}$ & $0.86^{*}(0.61$ to 1.20$)$ & 0.37 \\
\hline $\begin{array}{l}\text { Cox regression (time to atrial } \\
\text { fibrillation) }\end{array}$ & Intention to screen & $144 / 8874$ & $139 / 9102$ & $1.06+(0.84$ to 1.34$)$ & 0.61 \\
\hline $\begin{array}{l}\text { Cox regression (time to atrial } \\
\text { fibrillation) }\end{array}$ & Per protocol & $48 / 4085$ & $139 / 9102$ & $0.86+(0.62$ to 1.20$)$ & 0.38 \\
\hline
\end{tabular}

$\mathrm{N} / \mathrm{A}=$ not available.

Intention-to-screen analyses were adjusted for stratification variables (prevalence of atrial fibrillation and region). Per protocol analyses were also adjusted for age (in years), sex (male or female), and history of hypertension, diabetes mellitus, stroke (transient ischaemic attack or stroke), thromboembolism, and heart failure. Although a random intercept was included to adjust for clustering of patients in a care practice, the estimated intraclass correlation was o. For multiple imputation, we imputed the outcome with group, age, sex, and stratification variables.

* Odds ratio. HHazard ratio. 


\section{Atrial fibrillation detected with the screening protocol}

In the intention-to-screen group, 4106 of 9218 patients $(44.5 \%)$ participated in the screening protocol. The percentage of screened patients varied between practices, ranging from $6.7 \%$ to $65.8 \%$. Two practices discontinued screening during the study year because of organisational issues; both were included in the analysis.

Of 488 patients with at least one positive index test, 12 lead ECG was performed in 448 patients (fig 2). The median time between the first index test and 12 lead ECG was 26 minutes. In 40 patients, no electrocardiogram was obtained because of technical or organisational issues, or refusal of the patient. Also, ECG was performed in $294(8.1 \%)$ of 3616 patients with three negative index tests. Of 4106 patients screened, we found 26 patients with atrial fibrillation diagnosed by 12 lead ECG (0.63\%), all of whom had at least one positive index test. For patients with a negative electrocardiogram, 266 patients $(37.2 \%(266 / 716))$ continued with Holter monitoring for two weeks; four more new diagnoses of atrial fibrillation were detected $(1.50 \%(4 / 266))$.

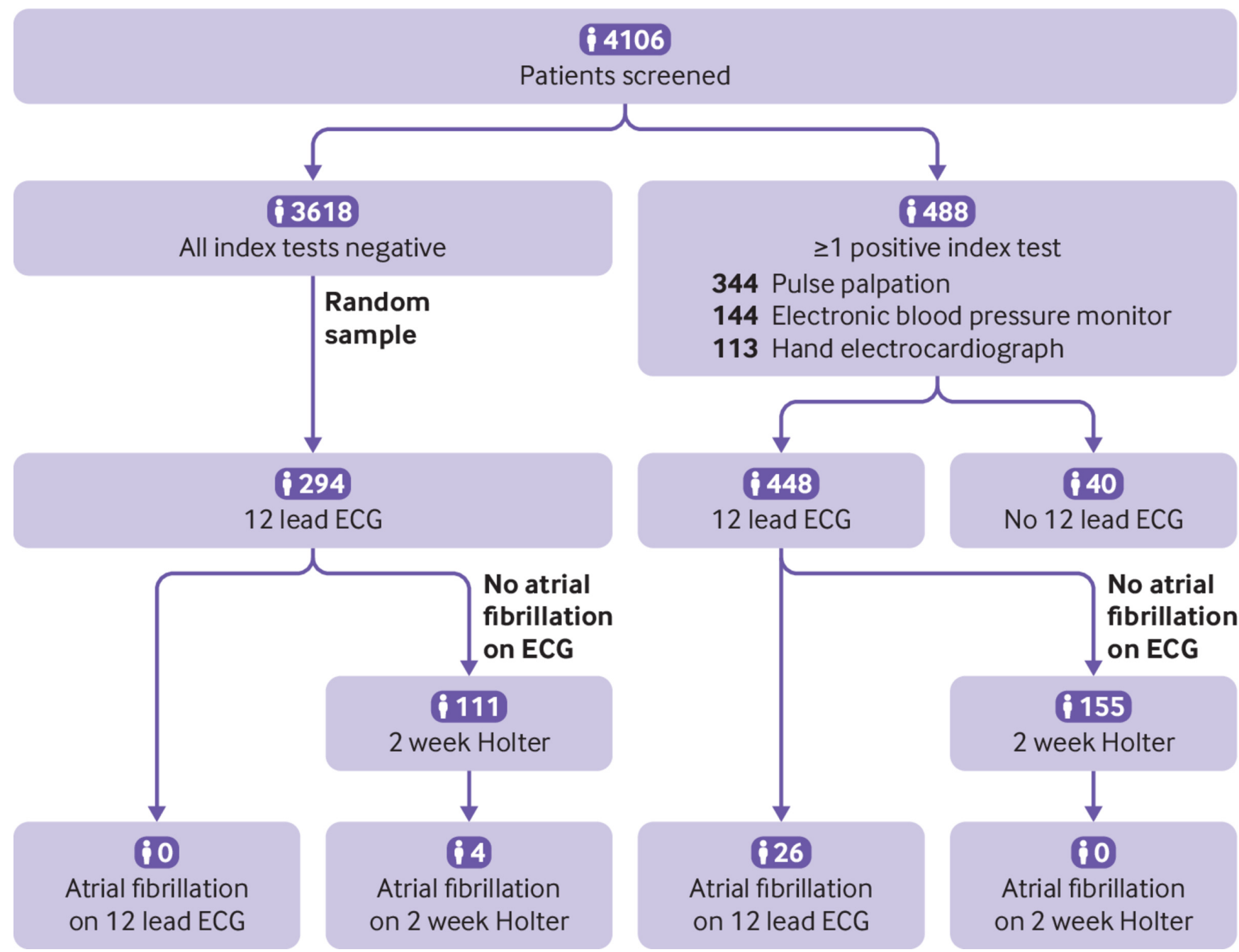

Fig 2 | Flowchart of the results of the screening intervention ( $n=4106)$. In 488 patients, electrocardiography (ECG) was required according to the protocol because the patient had at least one positive index test. An electrocardiogram was missing in 40 patients because of technical or organisational difficulties, or refusal of the patient. Of 3618 patients with three negative index tests, 294 were randomised to 12 lead ECG. Of 294 (with three negative index tests) and 422 (with at least one positive index test) patients who had a negative electrocardiogram, 266 continued with Holter monitoring for two weeks

Most patients with atrial fibrillation detected by screening were men. Six men had a CHA2DS2-VASc score of one (table 3). All other patients had a score of two or more. Patients with atrial fibrillation detected by screening were younger than those found during usual care in both the intention-to-screen and usual care practices (table 3). 
Table 3 | Characteristics of patients with newly diagnosed atrial fibrillation

\begin{tabular}{|c|c|c|c|c|c|c|}
\hline & \multicolumn{2}{|c|}{ Intention to screen } & \multicolumn{2}{|c|}{ Usual care } & \multicolumn{2}{|c|}{ Detected by one time point screening } \\
\hline & Female & Male & Female & Male & Female & Male \\
\hline No & 67 & 77 & 68 & 71 & 9 & 17 \\
\hline Age (mean (SD)) & $79.8(7.8)$ & $76.2(6.8)$ & $78.3(7.4)$ & $76.6(7.1)$ & $75.6(5.6)$ & $73.5(5.2)$ \\
\hline $65-75(\mathrm{No}(\%))$ & $23(34.3)$ & $36(46.8)$ & $27(39.7)$ & $31(43.7)$ & $5(55.6)$ & $10(58.8)$ \\
\hline $75-85($ No (\%)) & $22(32.8)$ & $34(44.2)$ & $25(36.8)$ & $30(42.3)$ & $3(33.3)$ & $7(41.2)$ \\
\hline$>85($ No (\%)) & $22(32.8)$ & $7(9.1)$ & $16(23.5)$ & $10(14.1)$ & $1(11.1)$ & 0 \\
\hline $\begin{array}{l}\text { Hypertension (No } \\
(\%) \text { ) }\end{array}$ & $49(73.1)$ & $40(51.9)$ & $47(69.1)$ & $31(43.7)$ & $6(66.7)$ & $8(47.1)$ \\
\hline $\begin{array}{l}\text { Stroke or transient } \\
\text { ischaemic attack } \\
\text { (No (\%)) }\end{array}$ & $9(13.4)$ & $11(14.3)$ & $15(22.1)$ & $7(9.9)$ & 0 & 0 \\
\hline Diabetes (No (\%)) & $15(22.4)$ & $20(26.0)$ & $21(30.9)$ & $16(22.5)$ & $1(11.1)$ & $2(11.8)$ \\
\hline $\begin{array}{l}\text { Heart failure (No } \\
(\%))\end{array}$ & $7(10.4)$ & $5(6.5)$ & $9(13.2)$ & $7(9.9)$ & $1(11.1)$ & $1(5.9)$ \\
\hline $\begin{array}{l}\text { Thromboembolism } \\
\text { (№ (\%)) }\end{array}$ & $4(6.0)$ & $5(6.5)$ & $5(7.4)$ & $2(2.8)$ & 0 & 0 \\
\hline \multicolumn{7}{|l|}{$\begin{array}{l}\text { CHA2DS2-VASC } \\
\text { score }(\text { No }(\%))^{*}\end{array}$} \\
\hline Score 1 & 0 & $10(13.0)$ & 0 & $5(7.0)$ & 0 & $6(35.3)$ \\
\hline Score 2 & $4(6.0)$ & $18(23.4)$ & $11(16.2)$ & $22(31.0)$ & $2(22.2)$ & $3(17.6)$ \\
\hline Score 3 & $21(31.3)$ & $21(27.3)$ & $13(19.1)$ & $21(29.6)$ & $2(22.2)$ & $5(29.4)$ \\
\hline Score 4 & $22(32.8)$ & $16(20.8)$ & $15(22.1)$ & $9(12.7)$ & $4(44.4)$ & $3(17.6)$ \\
\hline Score 5 & $8(11.9)$ & $6(7.8)$ & $15(22.1)$ & $10(14.1)$ & 0 & 0 \\
\hline Score $\geq 6$ & $12(17.9)$ & $6(7.8)$ & $14(20.5)$ & $4(5.6)$ & $1(11.1)$ & 0 \\
\hline \multicolumn{7}{|c|}{$\mathrm{SD}=$ standard deviation } \\
\hline
\end{tabular}

\section{Discussion}

In this cluster randomised controlled trial, opportunistic screening in primary care patients, aged 65 and over, did not increase the detection of previously unknown atrial fibrillation.

\section{Comparison with the literature}

Our results differed from the SAFE study where more diagnoses of atrial fibrillation were detected by screening than usual care $(1.63 \%$ $v 1.04 \%$, difference $=0.59 \%, 95 \%$ confidence interval 0.20 to 0.98$).{ }^{9}$ Our results are in line with the Improving Detection of Atrial Fibrillation in Primary Care with the MyDiagnostick (IDEAL-MD) trial, also conducted in the Netherlands, which did not detect more new diagnoses of atrial fibrillation by opportunistic screening than usual care $(1.43 \% \vee 1.37 \%, \mathrm{P}=0.73) .^{21}$

Why did we not detect more new diagnoses of atrial fibrillation with our extensive and sensitive screening protocol? One reason might be the younger age of the screened patients because the detection rate is dependent on the age of the population. ${ }^{22}$ Also, the baseline prevalence of atrial fibrillation in the participating centres (10.1\%) was higher than in the Rotterdam study (8.1\%) and the SAFE study (6.9-7.9\%), and much higher than the prevalence reported in a systematic review (4.4\%). ${ }^{2923}$ The high baseline prevalence of atrial fibrillation in our study strongly suggests that detection of atrial fibrillation is already high in usual care in the Netherlands. The high incidence of atrial fibrillation in the usual care practices $(1.53 \%$ $v 1.04 \%$ in the SAFE study) supports this view ${ }^{9}$ and might be explained by several reasons. Firstly, the introduction of the new oral anticoagulant agents in the past decade has raised awareness of the importance of timely detection of unknown atrial fibrillation. Secondly, most primary care practices in the Netherlands have introduced cardiovascular disease management programmes where the heart rhythm of patients is checked during visits. Thirdly, participating general practitioners might have had more interest in cardiovascular care than their non-participating colleagues. Finally, awareness of atrial fibrillation could have been raised by participating in a study about atrial fibrillation, the so-called Hawthorne effect. ${ }^{24}$ We tried to minimise this effect by blinding usual care practices to the selected patients in the practice.

Holter monitoring for two weeks detected a further $1.5 \%$ of new diagnoses of atrial fibrillation. Our findings showed that one time point screening missed silent and paroxysmal atrial fibrillation. Our results are in line with the Mass Screening for Untreated Atrial Fibrillation (STROKESTOP) and Akershus Cardiac Examination 1950 (ACE 1950) studies, which screened patients aged 75 and 65, respectively, with twice daily intermittent ECG for two weeks. ${ }^{25} 26$ In both studies, the detection rate at the index visit was $0.5 \%$. After two weeks of screening, a further $2.5 \%$ (in the STROKESTOP study) and $0.9 \%$ (in the ACE 1950 study) new diagnoses were detected. In the mHealth Screening to Prevent Strokes (mSToPS) trial (mean age 73.5), a two week monitoring period with a Holter patch without an index visit detected new atrial fibrillation in $4.7 \%$ of patients $(43 / 906) .^{27}$ 


\section{Strengths and weaknesses}

The intervention practices screened only $45 \%$ of eligible patients. This inclusion rate was higher than in the ThermoCool SmartTouch Catheter for the Treatment of Symptomatic Paroxysmal Atrial Fibrillation (AF-SMART) and IDEAL-MD studies, but lower than in the SAFE study. ${ }^{92128}$ We believe that the low inclusion rate was more likely because of organisational issues rather than patients not willing to participate. Despite our efforts to facilitate the study procedures, the study protocol was time consuming (three measurements, followed by ECG) and was executed during clinical hours in primary care practices with a high workload. We did not design the screening protocol with three index tests to implement in its entirety in primary care, however, but for maximum sensitivity and to establish the test characteristics of the new technologies. Future studies on screening procedures should aim to minimise the extra burden caused by the study design. Also, we selected a random sample of primary care patients, with no pre-selection by the physician, which increased the generalisability of our findings to the whole population, but also meant that a proportion of the selected patients were not eligible for screening because they could not visit the practice (eg, because they were frail) or for other reasons (eg, they had a pacemaker). The patients that participated in opportunistic screening were younger and had fewer comorbidities (so-called worried well) than patients who were not screened. Because the prevalence of atrial fibrillation increases with age, this might have led to an underestimation of the potential for screening when looking at the primary care population as a whole.

Although the percentage of screened patients was relatively low, the follow-up of a positive index test with 12 lead ECG was high compared with the follow-up in the SAFE study ( $92 \% v 66 \%$ ). In 40 patients with at least one positive index test, the required 12 lead ECG was missing. Visual inspection of the 40 single lead electrocardiogram recordings (after the end of the study and not part of the reference standard) did not suggest additional diagnoses of atrial fibrillation.

Unfortunately, only a small number of patients were willing to undergo Holter monitoring, possibly because of the inconvenience of wearing a device for two weeks. Our study was powered to evaluate opportunistic screening of patients visiting their general practitioner and the added diagnostic power of continuous screening was not taken into account in our power calculation.

\section{Implications and future research}

The results of our study are likely applicable to other well organised primary care populations outside of the Netherlands. In areas where a larger percentage of unknown atrial fibrillation is likely to be prevalent, opportunistic screening of atrial fibrillation might still be effective. Nevertheless, doctors' awareness of the importance of timely detection of atrial fibrillation is necessary. Future research on screening for atrial fibrillation should focus on the selection of patients with the highest risk of having or developing undetected atrial fibrillation and the role of repeated or prolonged monitoring. The uptake of continuous rhythm monitoring as a means of screening for atrial fibrillation might be more effective with new and wireless technologies, such as smart watches or wireless patches. $^{29}$

\section{Conclusion}

An extensive opportunistic screening protocol did not increase the detection of atrial fibrillation compared with usual care in patients aged 65 and over.

\section{What is already known on this topic}

- In 2007, the SAFE study showed that opportunistic screening for atrial fibrillation over a year detected more new diagnoses than usual care

- Randomised trials replicating the effectiveness of screening are lacking

- The effectiveness of prolonged screening on clinical endpoints, such as stroke and death, is not known

\section{What this study adds}

- In primary care, opportunistic screening in patients aged 65 and over did not lead to a higher detection rate of atrial fibrillation compared with usual care

Contributors: NV-vG and SBU contributed equally to this work. SBU, NV-vG, WAML, PMGE, JAK, HCPMvW, and HEJHS conceived and designed the study. JAK and HCPMvW supervised the study. WAML, PMGE, JAK, HCPMvW, and HEJHS obtained funding. SBU, NV-vG, WAML, BW, MN, and HEJHS acquired, analysed, and interpreted the data. SBU, NV-vG, and WAML wrote the first draft of the manuscript, and all authors revised the manuscript. SBU and NV-vG are the guarantors. The corresponding author attests that all listed authors meet authorship criteria and that no others meeting the criteria have been omitted.

Funding: This project was government funded by ZonMw (the Netherlands Organisation for Health Research and Development, grant No 839110006), and funded internally by Amsterdam Universities Medical Centres. Microlife/Retomed provided equipment without charge. Microlife/Retomed did not have a role in the study design, in the collection, analysis, and interpretation of the data, in the writing of the report, and in the decision to submit the article for publication.

Competing interests: All authors have completed the ICMJE uniform disclosure form at www.icmje.org/coi_disclosure.pdf and declare: support from the Netherlands Organisation for Health Research and Development and Amsterdam Universities Medical Centres for the submitted work; no financial relationships with any organisations that might have an interest in the submitted work in the previous three years; no other relationships or activities that could appear to have influenced the submitted work.

Ethical approval: The medical research ethics committee of the Amsterdam University Medical Centre (Amsterdam UMC), Amsterdam, approved the trial (14 November 2014, No NL48215.018.14). The D2AF study is registered at the Netherlands Trial Register (NTR reference No NL4776 (old NTR4914)).

Data sharing: Relevant anonymised patient level data are available on reasonable request.

The lead authors (SBU and NV-vG) affirm that this manuscript is an honest, accurate, and transparent account of the study being reported; that no important aspects of the study have been omitted; and that any discrepancies from the study as planned (and, if relevant, registered) have been explained.

Dissemination to participants and related patient and public communities: The project will lead to two $\mathrm{PhD}$ theses by general practitioners. The results have and will be presented at national and international conferences. Written reports will be published in international and national peer reviewed medical journals. Evidence from this study will be used in national and international guidelines on atrial fibrillation and cardiovascular risk management. The authors' network includes a co-author of the guideline for atrial fibrillation from the Dutch College of General Practitioners and a document reviewer for the 2010 European Society of Cardiology guideline on atrial fibrillation. HEJHS is part of the advisory board of the Staff Training Programme Cardiovascular Disease (Kaderopleiding Hart-en Vaatziekten) and a member of the GP Advisory Group Cardiovascular Diseases (Hart en Vaatziekten Huisartsen Adviesgroep, HartVaatHag). The D2AF study group is part of the AF Screen International Collaboration network. Thus the results will be distributed to vocational training programmes and continuing medical education courses in the Netherlands and abroad. Once the results are published, interest from newspapers and lay magazines is expected. The results of the study will be relevant for the Netherlands Heart Foundation (Nederlandse Hartstichting) and sharing of information through their website is expected, which is easily accessible for patients.

Provenance and peer review: Not commissioned; externally peer reviewed.

We thank Fysiologic ECG Services, Microlife/Retomed, MyDiagnostick Medical BV and MEMIC for their collaboration, in particular Esther Kuiper, Dave Hopman, Robert den Engelsman, Vincent Larik, Roger Hünen, Remon Winkel, Edwin Koekoek, Willem Verberk, Daniel Huysmans, and Dirk Veldman. Harry Crijns, Trang Dinh, Joris de Groot, Justin Luermans, and Maayke Sluman generously helped us in assessing the electrocardiograms. Piet Portegijs was our independent expert. We thank Alice Karsten, Ursula de Jonge Baas, and Frederique van Nouhuys of the Academic GP network Academic Medical Centre. Ralf Harskamp, Jelle Himmelreich, and Evert Karregat assisted the D2AF group. We also thank Mascha Twellaar, who assisted in data processing, and Sylvia de Graaf and Marion de Mooij, who were an essential part of the D2AF team. The study would not have been possible without the generous efforts of all 96 participating general practices and the selfless participation of all patients.

Kirchhof P, Benussi S, Kotecha D, etalESC Scientific Document Group. 2016 ESC Guidelines for the management of atrial fibrillation developed in collaboration with EACTS. Eur Heart J 2016;37:2893-962. doi: 10.1093/eurheartj/ehw210 pmid: 27567408 
2 Heeringa J, van der Kuip DA, Hofman A, etal. Prevalence, incidence and lifetime risk of atrial fibrillation: the Rotterdam study. Eur Heart /2006;27:949-53.

doi: 10.1093/eurhearti/ehi825 pmid: 16527828

3 Chugh SS, Roth GA, Gillum RF, Mensah GA. Global burden of atrial fibrillation in developed and developing nations. Glob Heart2014;9:113-9. doi: 10.1016/j.gheart.2014.01.004 pmid: 25432121

4 Marini C, De Santis F, Sacco S, etal. Contribution of atrial fibrillation to incidence and outcome of ischemic stroke: results from a population-based study. Stroke 2005;36:1115-9. doi: 10.1161/01.STR.0000166053.83476.4a pmid: 15879330

5 Zwietering PJ, Knottnerus JA, Rinkens PE, Kleijne MA, Gorgels AP. Arrhythmias in general practice: diagnostic value of patient characteristics, medical history and symptoms. Fam Pract 1998;15:343-53. doi: 10.1093/fampra/15.4.343 pmid: 9792350

6 Roche F, Gaspoz JM, Da Costa A, etal. Frequent and prolonged asymptomatic episodes of paroxysmal atrial fibrillation revealed by automatic long-term event recorders in patients with a negative 24-hour Holter. Pacing Clin Electrophysiol 2002;25:1587-93. doi: 10.1046/j.1460-9592.2002.01587.x pmid: 12494616

7 Lown M, Moran P. Should we screen for atrial fibrillation?BMJ2019;364:143. doi: 10.1136/bmj.l43 pmid: 30760476

8 Berge T. Wilson and Jungner would not approve of screening for atrial fibrillation. BMJ 2019;365:11416. doi: 10.1136/bmj.l1416 pmid: 31018937

9 Fitzmaurice DA, Hobbs FD, Jowett S, etal. Screening versus routine practice in detection of atria fibrillation in patients aged 65 or over: cluster randomised controlled trial. BMJ2007;335:383. doi: 10.1136/bmj.39280.660567.55 pmid: 17673732

10 Freedman B, Camm J, Calkins H, etalAF-Screen Collaborators. Screening for atrial fibrillation: a report of the AF-SCREEN International Collaboration. Circulation 2017;135:1851-67. doi: 10.1161/CIRCULATIONAHA.116.026693 pmid: 28483832

11 Uittenbogaart SB, Verbiest-van Gurp N, Erkens PM, etal. Detecting and diagnosing atrial fibrillation (D2AF): study protocol for a cluster randomised controlled trial. Trials 2015;16:478. doi: 10.1186/s13063-015-1006-5 pmid: 26499449

12 Netherlands Institute for Health Services Research. Nivel Primary Care Database: NIVEL.https://www.nivel.nl/en/nivel-primary-care-database. 2020

13 Nielen MMJ, Spronk I, Davids R, etal. Estimating morbidity rates based on routine electronic health records in primary care: observational study. JMIR Med Inform 2019;7: doi: 10.2196/11929 pmid: 31350839

14 Dutch College of General Practitioners. The practice guideline atrial fibrillation. Huisarts Wet 2013;8:392-401.

15 Dutch College of General Practitioners. The practice guideline cardiovascular risk management. Huisarts Wet 2012;1:14-28

16 Akker M, Metsemakers J, Limonard C, etal. General practice: a gold mine for research. Data and Scientific Use of the Registration Network Family Practices Maastricht. Maastricht University, 2004.

17 Dean AG, Sullivan KM, Soe MM. OpenEpi: Open source epidemiologic statistics for public health, version 3.03. www.OpenEpi.com. 2014.

18 Kelsey JL. Methods in observational epidemiology. Oxford University Press, 1996.

19 Smeeth L, Ng ES. Intraclass correlation coefficients for cluster randomized trials in primary care: data from the MRC Trial of the Assessment and Management of Older People in the Community. Control Clin Trials 2002;23:409-21. doi: 10.1016/S0197-2456(02)00208-8 pmid: 12161083

20 Campbell MK, Thomson S, Ramsay CR, MacLennan GS, Grimshaw JM. Sample size calculator for cluster randomized trials. Comput Biol Med 2004;34:113-25 doi: 10.1016/S0010-4825(03)00039-8 pmid: 14972631

21 Kaasenbrood F, Hollander M, de Bruijn SHM, etal. Opportunistic screening versus usual care for diagnosing atrial fibrillation in general practice: a cluster randomised controlled trial. Br J Gen Pract 2020;70:e427-33. doi: 10.3399/bjgp20X708161. pmid: 31988084

22 Lowres N, Olivier J, Chao T-F, etal. Estimated stroke risk, yield, and number needed to screen for atrial fibrillation detected through single time screening: a multicountry patient-level meta-analysis of 141,220 screened individuals. PLoS Med 2019;16:e1002903-03. doi: 10.1371/journal.pmed.1002903 pmid: 31553733

23 Lowres N, Neubeck L, Redfern J, Freedman SB. Screening to identify unknown atrial fibrillation. A systematic review. Thromb Haemost 2013;110:213-22. doi: 10.1160/TH13-02-0165 pmid: 23595785

24 McCarney R, Warner J, lliffe S, van Haselen R, Griffin M, Fisher P. The Hawthorne effect: a randomised, controlled trial. BMC Med Res Methodol 2007;7:30. doi: 10.1186/1471-2288-7-30 pmid: 17608932

25 Svennberg E, Engdahl J, Al-Khalili F, Friberg L, Frykman V, Rosenqvist M. Mass screening for untreated atrial fibrillation: the STROKESTOP study. Circulation 2015;131:2176-84. doi: 10.1161/CIRCULATIONAHA.114.014343 pmid: 25910800

26 Berge T, Brynildsen J, Larssen HKN, etal. Systematic screening for atrial fibrillation in a 65-year-old population with risk factors for stroke: data from the Akershus Cardiac Examination 1950 study. Europace 2018;20(FI_3):f299-305. doi: 10.1093/europace/eux293 pmid: 29095966

27 Steinhubl SR, Waalen J, Edwards AM, etal. Effect of a home-based wearable continuous ECG monitoring patch on detection of undiagnosed atrial fibrillation: the mSToPS randomized clinical trial. JAMA 2018;320:146-55. doi: 10.1001/jama.2018.8102 pmid: 29998336

28 Orchard J, Li J, Gallagher R, Freedman B, Lowres N, Neubeck L. Uptake of a primary care atrial fibrillation screening program (AF-SMART): a realist evaluation of implementation in metropolitan and rural general practice. BMC Fam Pract 2019;20:170

doi: 10.1186/s12875-019-1058-9 pmid: 31810441
29 Perez MV, Mahaffey KW, Hedlin H, etalApple Heart Study Investigators. Large-scale assessment of a smartwatch to identify atrial fibrillation. N Engl J Med 2019;381:1909-17. doi: 10.1056/NEJMoa1901183 pmid: 31722151

This is an Open Access article distributed in accordance with the Creative Commons Attribution Non Commercial (CC BY-NC 4.0) license, which permits others to distribute, remix, adapt, build upon this work non-commercially, and license their derivative works on different terms, provided the original work is properly cited and the use is non-commercial. See: http://creativecommons.org/licenses/by$\mathrm{nc} / 4.0 \%$. 